\title{
Coupled Transmission Lines as Impedance Transformer
}

\author{
Jensen, Thomas; Zhurbenko, Vitaliy; Krozer, Viktor; Meincke, Peter
}

Published in:

IEEE Transactions on Microwave Theory and Techniques

Link to article, DOI:

10.1109/TMTT.2007.909617

Publication date:

2007

Document Version

Peer reviewed version

Link back to DTU Orbit

Citation (APA):

Jensen, T., Zhurbenko, V., Krozer, V., \& Meincke, P. (2007). Coupled Transmission Lines as Impedance Transformer. IEEE Transactions on Microwave Theory and Techniques, 55(12), 2957-2965.

https://doi.org/10.1109/TMTT.2007.909617

\section{General rights}

Copyright and moral rights for the publications made accessible in the public portal are retained by the authors and/or other copyright owners and it is a condition of accessing publications that users recognise and abide by the legal requirements associated with these rights.

- Users may download and print one copy of any publication from the public portal for the purpose of private study or research.

- You may not further distribute the material or use it for any profit-making activity or commercial gain

- You may freely distribute the URL identifying the publication in the public portal

If you believe that this document breaches copyright please contact us providing details, and we will remove access to the work immediately and investigate your claim 


\title{
Coupled Transmission Lines as Impedance Transformer
}

\author{
Thomas Jensen, Vitaliy Zhurbenko, Viktor Krozer, Peter Meincke \\ Technical University of Denmark, Ørsted $\cdot D T U$, ElectroScience, \\ Ørsteds Plads, Building 348, 2800 Kgs. Lyngby, Denmark, Phone:+45-45253861, Fax: +45-45931634, \\ E-mail: tje@oersted.dtu.dk
}

\begin{abstract}
A theoretical investigation of the use of a coupled line section as an impedance transformer is presented. It is shown how to properly select the terminations of the coupled line structures for effective matching of real and complex loads in both a narrow and a wide frequency range. The corresponding circuit configurations and the design procedures are proposed. Synthesis relations are derived and provided for efficient matching circuit construction. Design examples are given to demonstrate the flexibility and limitations of the design methods and to show their validity for practical applications. Wideband matching performance with relative bandwidth beyond $100 \%$ and return loss $R L>20 \mathrm{~dB}$ is demonstrated both theoretically and experimentally. Good agreement is achieved between the measured and predicted performance of the coupled line transformer section.

Index Terms - Coupled transmission lines, directional coupler, impedance matching, impedance matrix, microstrip lines, strip lines.
\end{abstract}

\section{INTRODUCTION}

I $\mathrm{n}$ recent years, coupled transmission lines have been 1 suggested as a matching element due to greater flexibility and compactness in comparison to quarter wavelength transmission lines [1-3]. It has been demonstrated that matching real and complex loads with coupled lines leads to more compact realizations and could therefore become important at millimeter-wave frequencies for on-chip or Low Temperature Cofired Ceramics (LTCC) matching solutions. Another area where coupled line structures are useful is matching of antenna array structures, as successfully demonstrated in [2].

The quarter-wave transformer is simple and easy to use, but it has no flexibility beyond the ability to provide a perfect match at the center frequency for a real-valued load, although a complex load of course can be matched by increasing the length of the quarter-wave transformer. The coupled line section provides a number of variables which can be utilized for matching purposes. These variables are the even and odd mode impedances and loading of the through and coupled ports. This loading can be done in form of a feedback connection which provides additional zeros for broadband matching.

These variables can be chosen to provide a perfect match or any desired value of the reflection coefficient at the operating frequency. The bandwidth of the coupled line transformer can be further increased in case of mismatch. In addition, as will be shown, it is also possible to match a complex load. This is a generalization of matching with a quarter-wave transmission line transformer.

This paper focuses on developing the necessary formulas for applying coupled line sections in matching applications, as well as the appropriate basic analysis of the coupled line section. Establishing a design framework will enable a widespread use of coupled line structures as novel impedance matching elements, in addition to the standard lines and lumped elements.

In the lower $\mathrm{GHz}$ range the loading of the through and coupled ports can be done with lumped elements which allows for easy matching of both real and imaginary impedance values. At higher frequencies it is not possible to use lumped elements, but the difference between the even and odd mode impedances is a parameter which makes it possible to turn a mixed real and imaginary control load at the through port into a purely imaginary one, which can be implemented with a transmission line stub.

Equations for matching purposes, which are based on controllable parameters of coupled transmission line sections, are presented for backward-wave couplers including microstrip and stripline transmission line couplers.

The coupling required for a given application often becomes too tight for a practical implementation. Therefore an investigation into the range of load values that can realistically be matched with the coupled line section has been carried out.

Finally loading of the through and coupled port with an interconnecting transmission line is considered with the purpose to achieve a wide operating frequency range. In [5] a broadband impedance transformer based on coupled transmission lines is presented. The synthesis procedure for this circuit is explained. By using this procedure it is possible to shape the frequency response by placing transmission minima in the spectrum.

\section{THE USE OF A COUPLED LINE SECTION AS AN IMPEDANCE TRANSFORMER}

Fig. 1 shows the general coupled lines configuration. There is no established terminology for use of the coupled line section as an impedance transformer, in this paper it is found useful to use the port names from directional coupler terminology in the discussion of the circuit, but the numeration used corresponds to filter design with coupled line sections because the theory is developed from that point of view. 


\section{Coupled Lines}

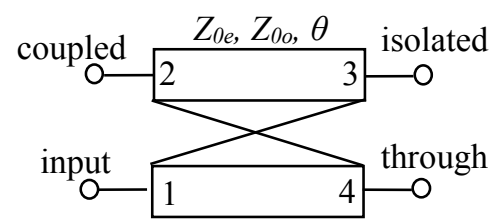

Fig. 1. Coupled transmission lines. The port names are in agreement with directional coupler terminology for a backward-wave coupler, while the numeration is the same as used in filter design with coupled line sections.

The impedance matrix for the 4-port open-circuited coupled line section in Fig. 1, where transverse electromagnetic (TEM) wave propagation and symmetric transmission lines are assumed, can be expressed in terms of the even and odd mode impedances, $Z_{0 e}$ and $Z_{0 o}$, and the electrical length, $\theta[4]$ :

$$
\begin{aligned}
& Z_{11}=Z_{22}=Z_{33}=Z_{44}=-j / 2\left(Z_{0 e}+Z_{0 o}\right) \cot \theta, \\
& Z_{12}=Z_{21}=Z_{34}=Z_{43}=-j / 2\left(Z_{0 e}-Z_{0 o}\right) \cot \theta, \\
& Z_{13}=Z_{31}=Z_{24}=Z_{42}=-j / 2\left(Z_{0 e}-Z_{0 o}\right) \csc \theta, \\
& Z_{14}=Z_{41}=Z_{23}=Z_{32}=-j / 2\left(Z_{0 e}+Z_{0 o}\right) \csc \theta,
\end{aligned}
$$

$j=\sqrt{-1}$.

The input impedance of the coupled line section with the coupled and through port open-circuited and the isolated port terminated in the load impedance $Z_{L}$, can be calculated from the current and voltage at the input port

$$
Z_{I N}=\frac{V_{1}}{I_{1}}=\frac{Z_{11} Z_{L}+|Z|}{Z_{L}+Z_{22}},
$$

where $|Z|=Z_{11} Z_{22}-Z_{21} Z_{12}$.

In the following derivations only the center frequency is considered and hence, $\theta=\pi / 2, \cot \theta=0$, and $\csc \theta=1$. Furthermore the notation $Z_{+}=Z_{0 e}+Z_{0 o}$ and $Z_{-}=Z_{0 e}-Z_{0 o}$ is used. Then (2) simplifies to

$$
Z_{I N}=\frac{-Z_{21} Z_{12}}{Z_{L}}=\frac{-(-j / 2)^{2} Z_{-}^{2}}{Z_{L}}=\frac{Z_{-}^{2}}{4 Z_{L}} .
$$

The reflection coefficient with the generator impedance $Z_{g}$ attached to port 1 is given by

$$
\Gamma_{I N}=\frac{Z_{I N}-Z_{g}}{Z_{I N}+Z_{g}}=\frac{\frac{Z_{-}^{2}}{4 Z_{L}}-Z_{g}}{\frac{Z_{-}^{2}}{4 Z_{L}}+Z_{g}} .
$$

For a perfect match the reflection coefficient has to be zero and thus

$$
\frac{Z_{-}^{2}}{4 Z_{L}}-Z_{g}=0
$$

Solving for $Z$. leads to

$$
Z_{0 e}-Z_{0 o}=2 \sqrt{Z_{g} Z_{L}}
$$

This establishes the condition for a perfect match at the operating frequency in terms of the difference between the even and odd mode impedance. This condition is similar to the well-known relation $Z_{0}=\sqrt{Z_{g} Z_{L}}$, for a quarter-wave transmission line transformer, when the characteristic impedance is exchanged with the difference between the even and odd mode impedances of the coupled lines.

It is also possible to retain the reflection coefficient in the formula and use it as a parameter, yielding

$$
Z_{0 e}-Z_{0 o}=2 \sqrt{Z_{g} Z_{L} \frac{1+\Gamma_{I N}}{1-\Gamma_{I N}}} .
$$

The parameter $Z$. can be chosen within reasonable limits. A large value of $Z$. implies a tight coupling, where the coupling coefficient is given by $C=\frac{Z_{0 e}-Z_{0 o}}{Z_{0 e}+Z_{0 o}}$. Tight coupling implies a large value of $\mathrm{C}$ and requires a large difference between the even and odd mode impedances. A large value of $Z$. necessitates a very narrow gap between edge-coupled transmission lines or a large ground plane spacing, e.g. in stripline technology.

From (7) it can be seen that transforming a large-valued load requires a tighter coupling than a small-valued load, and even though a non-zero reflection coefficient can increase the bandwidth it also requires tighter coupling.

The quarter-wave transformer has the same length as the coupled line impedance transformer for a given application and requires only one transmission line, so the only obvious advantage of the latter is that it constitutes a perfect DC block. However, a load attached to the through port opens for improvement of the transformer characteristics for the coupled line section as will be shown in the next sections.

\section{A. Loading of the through port for extended matching capabilities.}

Using the input port as input and with the load to be transformed attached to the isolated port leaves two available ports (the through and coupled port) that can be exploited to improve the matching capabilities. The loading of the through port will be considered in this section.

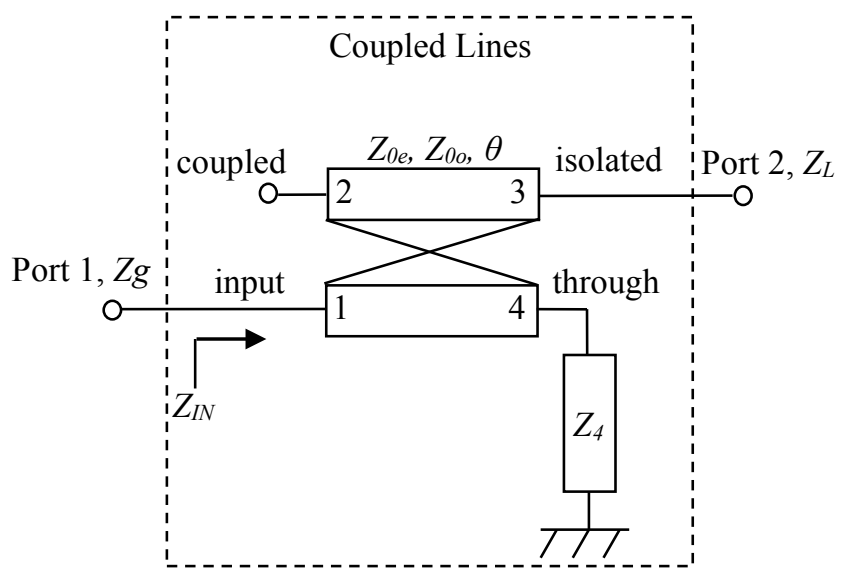

Fig. 2. The 4-port coupled transmission line section reduced to a 2-port with the coupled port open-circuited and the through port terminated in load $Z_{4}$. 
The open-circuited impedance matrix $[Z]$ for a two-port that is based on the four-port coupled line section in Fig. 2, where the coupled port is left open-circuited and the through port is loaded with an arbitrary impedance $Z_{4}$, that will be used as a matching parameter, can be derived as (see appendix II)

$$
[Z]=\left[\begin{array}{cc}
\frac{Z_{+}^{2}}{4 Z_{4}} & \frac{-j}{2} Z_{-} \\
\frac{-j}{2} Z_{-} & 0
\end{array}\right] .
$$

The impedance matrix is symmetric and therefore the circuit is reciprocal, but $Z_{4}$ should be chosen imaginary or with a large real part to avoid excessive power dissipation. The input impedance derived from (2) is then

$$
Z_{I N}=\frac{Z_{+}^{2}}{4 Z_{4}}+\frac{Z_{-}^{2}}{4 Z_{L}} .
$$

Substituting this expression in the formula for the input reflection coefficient (4) and solving for $Z_{4}$ gives

$$
Z_{4}=\frac{Z_{+}^{2}\left(1-\Gamma_{I N}\right)}{4 Z_{g}\left(1+\Gamma_{I N}\right)-\frac{Z_{-}^{2}}{Z_{L}}\left(1-\Gamma_{I N}\right)},
$$

which determines the value of $Z_{4}$ for a given value of $Z_{g}, Z_{L}$, $Z_{+}, Z_{\text {., and }} \Gamma_{\mathrm{IN}}$. The condition for $Z_{4}=\infty$, i.e. open-circuited, is only fulfilled for $Z_{-}=2 \sqrt{Z_{g} Z_{L} \gamma}$. This demonstrates that the coupled line section impedance transformer is indeed a generalization of the standard transmission line transformer, where the impedance $Z_{4}$ represents an additional degree of freedom.

\section{Matching example with a real-valued load}

The ability of the coupled line section to match a realvalued load is illustrated with a simple example.

The following parameters are given: $Z_{g}=50 \Omega, Z_{L}=100 \Omega$, $Z_{+}=200 \Omega, Z_{-}=100 \Omega$, and $\Gamma_{I N}=0.032$.

Using (10) results in $Z_{4}=353 \Omega$. The circuit configuration is shown in Fig. 3 and the frequency response plotted at an arbitrary design frequency of $1 \mathrm{GHz}$ in Fig. 4.

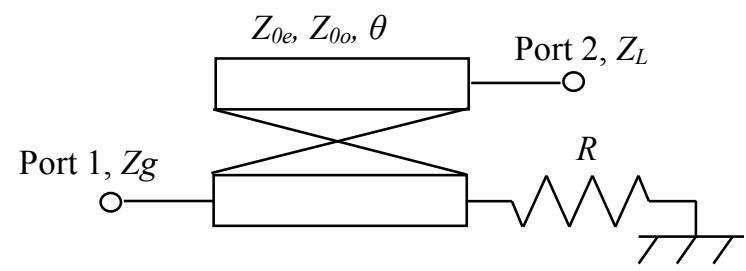

Fig. 3. Matching configuration example. The response with $Z_{g}=50 \Omega, Z_{L}=$ $100 \Omega, Z_{+}=200 \Omega$, and $Z_{-}=100 \Omega$ is shown in Fig. 4.

A reflection coefficient of 0.032 corresponds to $-30 \mathrm{~dB}$. In this example a reflection coefficient magnitude $<-20 \mathrm{~dB}$ spans a bandwidth of $22 \%$.

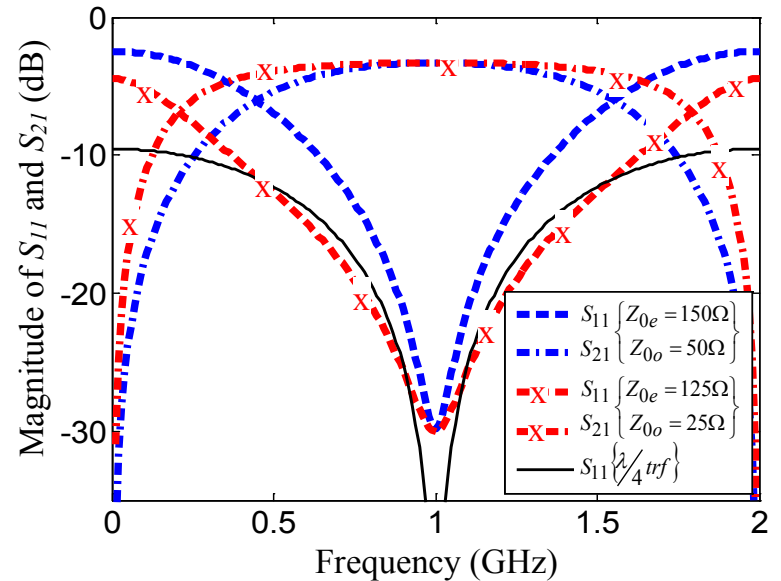

Fig. 4. Response of the circuit in Fig. 3. $\left|\mathrm{S}_{11}\right|$ is $-30 \mathrm{~dB}$ at the design frequency as desired. Note that the insertion loss is non-zero because power is dissipated in the resistor. The response with tighter coupling $\left(Z_{0_{e}}=125 \Omega, Z_{0_{o}}=25 \Omega\right)$ and a comparision with a quarter-wave transformer is also included in the plot.

If the coupling is increased, setting $Z_{0 e}=125 \Omega$ and $Z_{0 o}=25 \Omega$, results in $Z_{4}=199 \Omega$ and an increased bandwidth of $44 \%$ for the same matching conditions. The physical dimensions for the first case in edge-coupled stripline technology with $\varepsilon_{\mathrm{r}}=3.38$ and a ground plane spacing of $5 \mathrm{~mm}$ are a conductor width of $0.55 \mathrm{~mm}$ and a conductor separation of $0.21 \mathrm{~mm}$.

\section{B. Achieving purely imaginary control loads}

An interesting property of the coupled line section as a matching element is the ability to match a complex load. Splitting $Z_{4}$ in a real and an imaginary part gives

$$
\begin{aligned}
& Z_{4}=\frac{Z_{+}^{2}\left(4 Z_{g} \gamma\left|Z_{L}\right|^{2}-Z_{-}^{2} \operatorname{Re}\left\{Z_{L}\right\}\right)}{\Delta}-j \frac{Z_{-}^{2} Z_{+}^{2} \operatorname{Im}\left\{Z_{L}\right\}}{\Delta}, \\
& \Delta=16 Z_{g}^{2} \gamma^{2}\left|Z_{L}\right|^{2}+Z_{-}^{2}\left(Z_{-}^{2}-8 Z_{g} \gamma \operatorname{Re}\left\{Z_{L}\right\}\right),
\end{aligned}
$$

where $\gamma=\frac{1+\Gamma_{I N}}{1-\Gamma_{I N}}, Z_{g}$ and $\gamma$ are assumed real-valued.

From this expression it can be seen that it is possible to use $Z$. to make the real part of $Z_{4}$ equal to zero, which establishes a condition for a purely imaginary value of $Z_{4}$

$$
\begin{gathered}
Z_{+}^{2}\left(4 Z_{g} \gamma\left|Z_{L}\right|^{2}-Z_{-}^{2} \operatorname{Re}\left\{Z_{L}\right\}\right)=0, \\
Z_{-}=2\left|Z_{L}\right| \sqrt{\frac{Z_{g} \gamma}{\operatorname{Re}\left\{Z_{L}\right\}}} .
\end{gathered}
$$

This is especially useful for high frequency applications, where the matching load to be attached to $Z_{4}$ can simply be realized as a transmission line stub of a specified length. An added advantage is that a purely reactive load is in principle lossless.

\section{Matching example with a complex-valued load}

The following parameters are given: $Z_{g}=50 \Omega$, $Z_{L}=40+\mathrm{j} 20 \Omega, Z_{+}=200 \Omega$, and $\Gamma_{I N}=0$.

$Z$. is found to be $100 \Omega$ and $Z_{4}=-\mathrm{j} 400 \Omega$ which can be implemented as a short transmission line stub with a length of $12.1^{\circ}$ and a characteristic impedance of $\sqrt{Z_{0 e} Z_{0 o}}=86.6 \Omega$. 

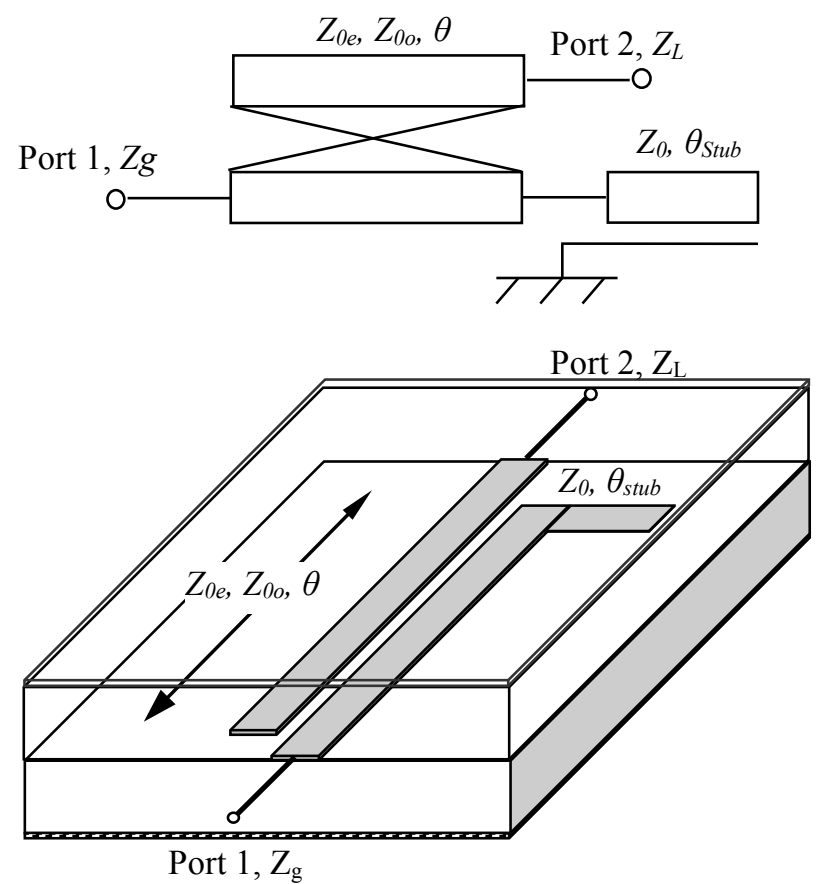

Fig. 5. Matching configuration for a complex load. Schematic drawing above and possible stripline realization below. The response with the parameters $Z_{g}$ $=50 \Omega, Z_{L}=40+\mathrm{j} 20 \Omega, Z_{+}=200 \Omega, Z_{-}=100 \Omega, Z_{0}=86.6 \Omega$, and $\theta_{\text {Stub }}=$ $12.1^{\circ}$ is shown in Fig. 6.

The characteristic impedance of the stub can be chosen to give a line width equal to the transmission lines in the coupled lines section in order to avoid discontinuities. Here $\sqrt{Z_{0 e} Z_{0 o}}$ is used for the stub line characteristic impedance, but it is not a necessary condition.

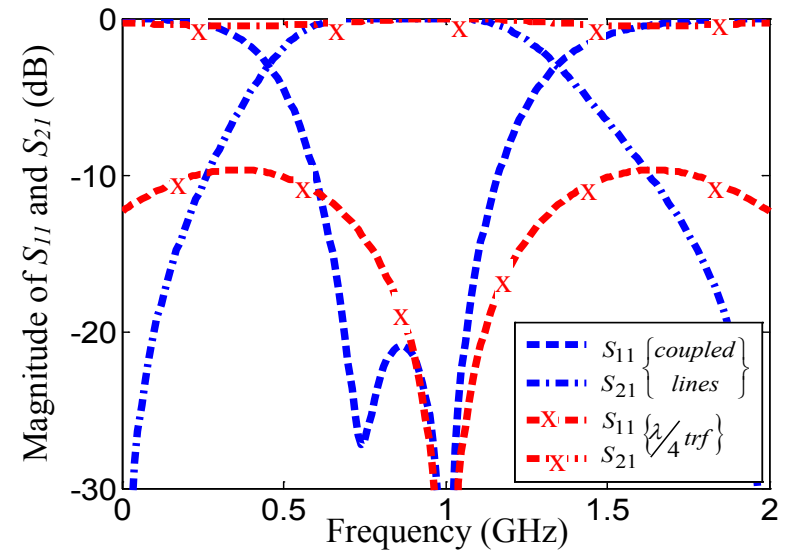

Fig. 6. Response of the circuit in Fig. 5 and compared with a quarter-wave transformer. Note that this matching configuration is lossless because $Z_{4}$ is purely imaginary.

The response in Fig. 6 shows a perfect match at the center frequency and a $-20 \mathrm{~dB}$ bandwidth of $31 \%$. The length of the matching stub is very short compared to traditional transmission line matching circuits and will only slightly increase the circuit size.

The load used in this example can also be matched with a quarter-wave transformer and an extra length of transmission line to account for the imaginary part of the load, but the bandwidth achieved is smaller and out of band rejection is significantly worse, see Fig. 6 .

The limitation of the coupled line section becomes apparent when $Z_{4}$ from (10) is plotted against a range of possible load values. Fig. 7 shows the required value of $Z_{4}$ for a perfect match plotted versus the real part of $Z_{L}$. The figure depicts two sets of curves, one for the real part of $Z_{4}$ and one for the imaginary part of $Z_{4}$, for imaginary load values, $\operatorname{Im}\left\{Z_{L}\right\}$ of 20 , 40 and $60 \Omega$, respectively, $Z_{0 e}=125 \Omega, Z_{0 o}=25 \Omega$, and $Z_{g}=$ $50 \Omega$.

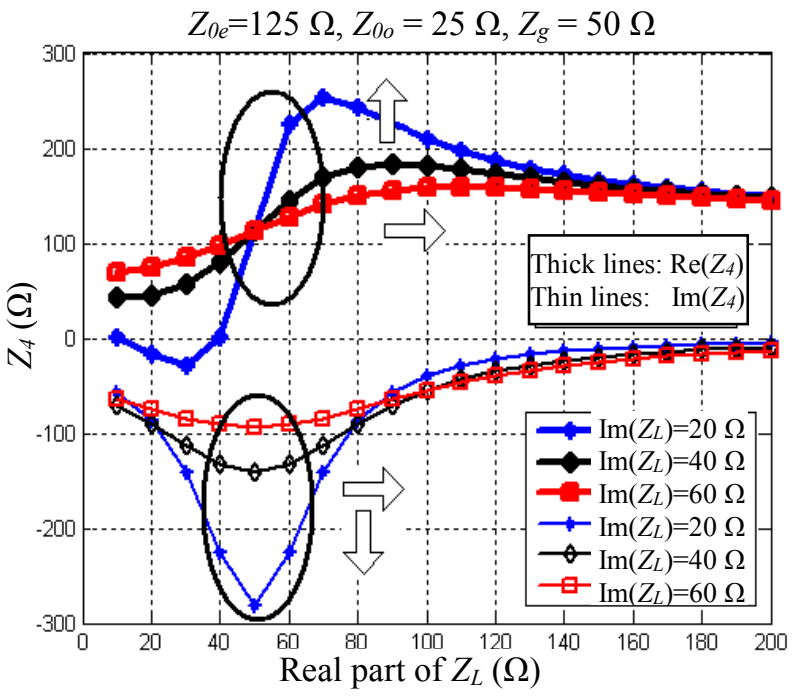

Fig. 7. The arrows indicate the general direction of the curves when the coupling is increased, i.e. the required value of the real part of $Z_{4}$ becomes more positive and the imaginary part becomes more negative. Note that for this choice of the even and odd impedance, a negative real part is required for $Z_{4}$ when $\operatorname{Im}\left\{Z_{L}\right\}=20 \Omega$ and $\operatorname{Re}\left\{Z_{L}\right\}<40 \Omega$ (thick blue curve below 0 ).

To decrease the power dissipation a large value of $Z_{4}$ is desirable, but this implies stronger coupling. Decreasing the coupling will lower the real part of $Z_{4}$ towards the value of $Z_{g}$ with the result that most of the input power is dissipated in $Z_{4}$.

If the condition for a purely imaginary value of $Z_{4}$, Eq. (13), is applied then Fig. 8 shows that tight coupling values are required for large-valued loads.

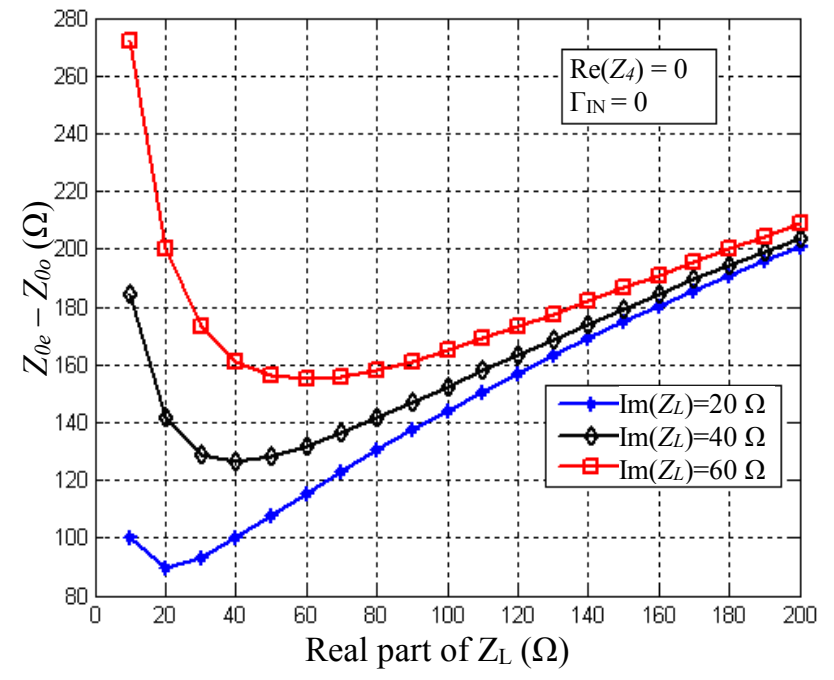

Fig. 8. Values of $Z_{0 e}-Z_{0 o}$ when $Z_{4}$ is required to be purely imaginary and $\Gamma_{\mathrm{IN}}=0$ (perfect match). 
It can be depicted that very tight coupling is required for even moderate impedance loads. Relaxing the condition for the reflection coefficient, $\Gamma_{I N}$, moves the curves towards higher values and will therefore require even tighter coupling.

A coupling coefficient larger than $-10 \mathrm{~dB}$ is difficult to achieve with microstrip or stripline edge-coupled lines, but is possible with a thick substrate or with broadside coupled transmission lines.

\section{WIDEBAND IMPEDANCE TRANSFORMATION.}

\section{A. Derivation of analysis formulas}

The impedance transformers considered above are based on transmission lines in homogeneous medium, e.g. striplines. They allow for a simpler analysis, however, in many practical cases, for example in surface mount technology, it is more useful to deal with microstrip structures.

The wideband impedance transformer proposed in this section is derived on the basis of asymmetric, uniformly coupled lines in a nonhomogeneous medium [5]. A microstrip line is one of the most commonly used classes of transmission lines in a nonhomogeneous medium. The proposed configuration is a quarter wavelength long and provides three times wider operating frequency range in comparison to the traditional quarter-wave transformer.

As discussed above the matching properties of the transformer depend not only on coupled line parameters, but also on the loads at ports 2 and 4 in Fig. 2. This dependence introduces additional degrees of freedom during the design procedure and is used here to significantly expand the bandwidth of the impedance transformer. The configurations shown above use loading of terminal 4 and with terminal 2 open-circuited. In the circuit considered below, both terminals are loaded using an interconnecting microstrip stepped impedance transmission line as shown in Fig. 9.
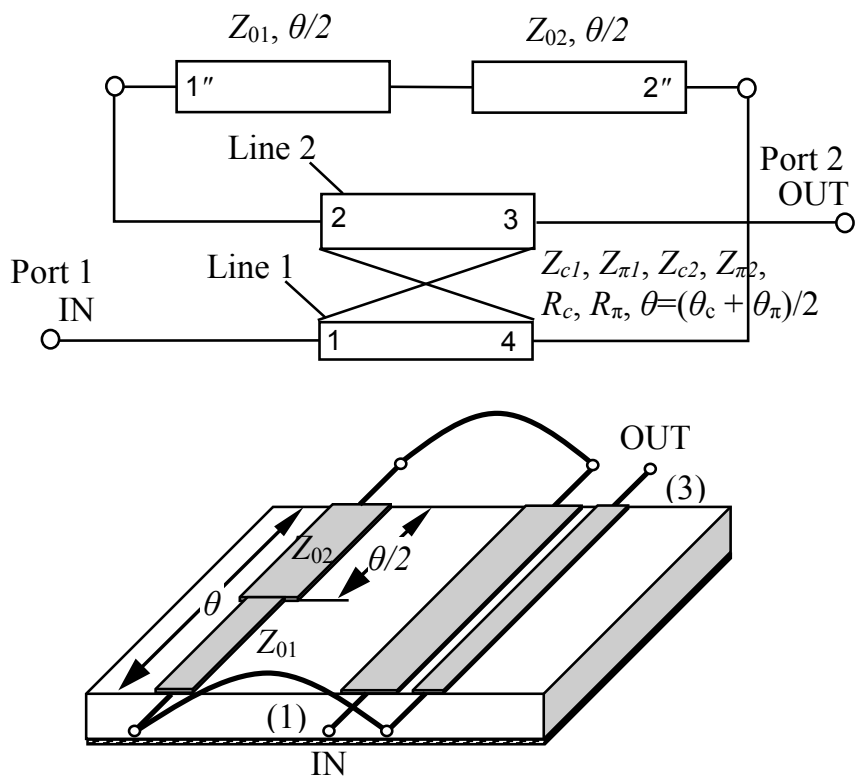

Fig.9. The quarter-wave wideband impedance transformer. Schematic drawing above and possible microstrip realization below.
The transformer consists of asymmetric coupled lines described by the electrical parameters $Z_{c l}, Z_{c 2}, Z_{\pi l}, Z_{\pi 2}$, which are, respectively, the characteristic impedances of line 1 and 2 for the $c$ and $\pi$ modes of propagation; $\theta_{c}$ and $\theta_{\pi}$, the electrical lengths for the $c$ and $\pi$ modes; $R_{c}$ and $R_{\pi}$, the ratios of the voltages on the two lines for the $c$ and $\pi$ modes. The stepped impedance transmission line consists of two equal length transmission lines with characteristic impedances $Z_{01}$ and $Z_{02}$, as shown in Fig. 9. The electrical length of each transmission line is set to be half the electrical length of the coupled line section to reduce the number of design parameters.

For the purpose of analysis, this structure is transformed to a two-port network with arbitrary load using an impedance matrix representation. Thus, the entire circuit can be represented as a two-port network, which performs impedance transformation between a generator impedance $Z_{g}$ connected to port 1 and a load impedance $Z_{L}$ connected to port 3, as shown in Fig. 10.

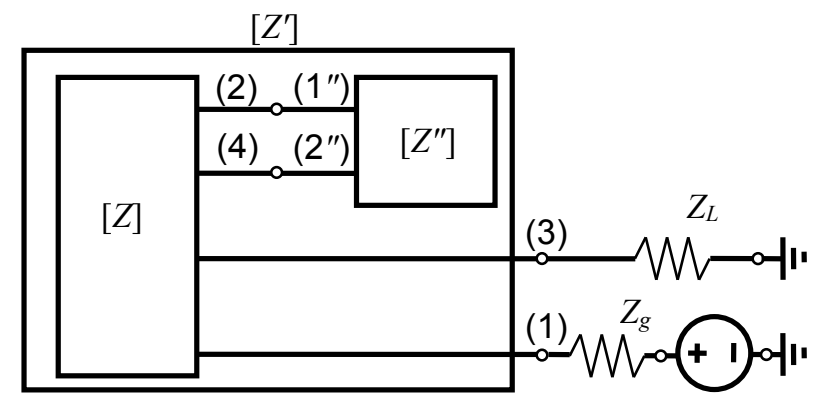

Fig. 10. Two-port network representation for the coupled line impedance transformer. From [5].

The model in Fig. 10 consists of the coupled line four-port network described by an impedance matrix $[Z]$ and arbitrary load matrix at opposite terminals described by matrix [ $\left.Z^{\prime \prime}\right]$. In practice, ports 2 and 4 are in general either short-circuited or open-circuited with a corresponding representation of the twoport network [Z"].

The magnitude of the reflection coefficient at port 1 is equal to

$$
\left|S_{11}\right|_{\mathrm{dB}}=20 \log \left(\left|\frac{Z_{I N}\left(Z_{i j}, Z_{i j}^{\prime \prime}, Z_{L}\right)-Z_{g}}{Z_{I N}\left(Z_{i j}, Z_{i j}^{\prime \prime}, Z_{L}\right)+Z_{g}}\right|\right),
$$

where $Z_{I N}$ is the input impedance of the transformer, which is a function of the load impedance $Z_{L}$, impedance matrix elements of coupled lines $Z_{\mathrm{ij}}$ and the arbitrary load $Z_{i j}^{\prime \prime}$ (i and $j$ are the indexes of matrix elements).

The input impedance $Z_{I N}$ is calculated using relations derived in [5] together with the following elements of the impedance matrix $\left[Z^{\prime \prime}\right]$ for the stepped impedance transmission line

$$
Z_{11}^{\prime \prime}=Z_{01} \operatorname{coth}\left(\frac{\theta}{2}\right)-\frac{2 Z_{01}^{2}}{\left(Z_{01}+Z_{02}\right) \cdot \sinh (\theta)}
$$




$$
\begin{aligned}
& Z_{12}^{\prime \prime}=Z_{21}^{\prime \prime}=\frac{2 Z_{01} Z_{02}}{\left(Z_{01}+Z_{02}\right) \cdot \sinh (\theta)}, \\
& Z_{22}^{\prime \prime}=Z_{02} \operatorname{coth}\left(\frac{\theta}{2}\right)-\frac{2 Z_{02}^{2}}{\left(Z_{01}+Z_{02}\right) \cdot \sinh (\theta)} .
\end{aligned}
$$

The derivation of (15) is given in Appendix I.

Thus, the analysis of the structure can be performed using this analytical representation.

The electrical length is a function of frequency and is used here for the analysis of the spectrum of the transformer reflection coefficient. It can be depicted from the calculated response in Fig. 11 that the transformer provides wideband operation, and the electrical length of the transformer is equal to a quarter wavelength at the center frequency.

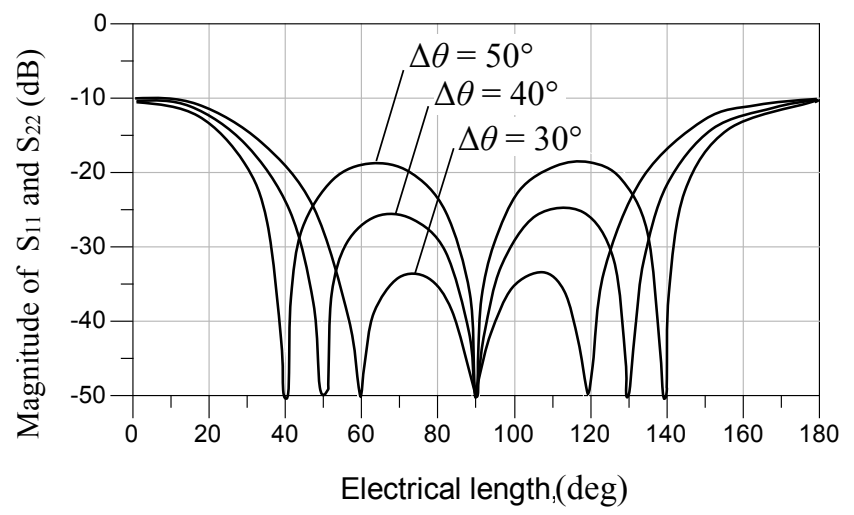

Fig. 11. Response of the 50-100 $\Omega$ impedance transformer shown in Fig. 9.

In addition, the distance between the minima locations $\Delta \theta$ can be varied by adjusting the parameters of the structure. This distance $\Delta \theta$ characterizes operating frequency bandwidth of the transformer. The characteristics of the transformer for three different values of $\Delta \theta$ are shown in Fig. 11. As it can be seen, the in-band level of the reflection coefficient depends on parameter $\Delta \theta$. The estimation of the maximum level of the reflection coefficient between minima for different transformation ratios can be found using the data shown in Fig. 12.

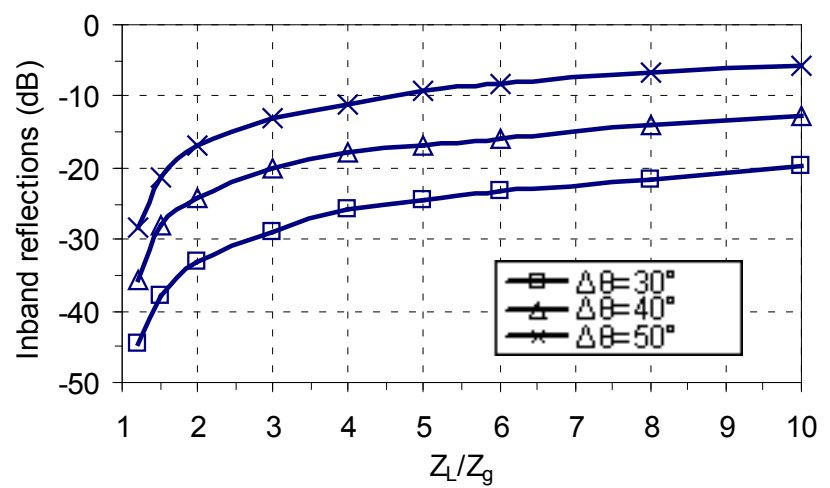

Fig. 12. The maximum level of the reflection coefficient between minima in Fig. 11.
As expected, the level of in-band reflections for the transformer decreases with reducing of transformation ratio, and reaches the absolute minimum at $Z_{L} / Z_{g}=1$.

\section{B. Synthesis of the wideband transformer}

Equation (14) was solved numerically for the transformer in Fig. 9 with respect to the design parameters. Based on these solutions, design curves for this transformer are obtained and shown in Fig. 13.
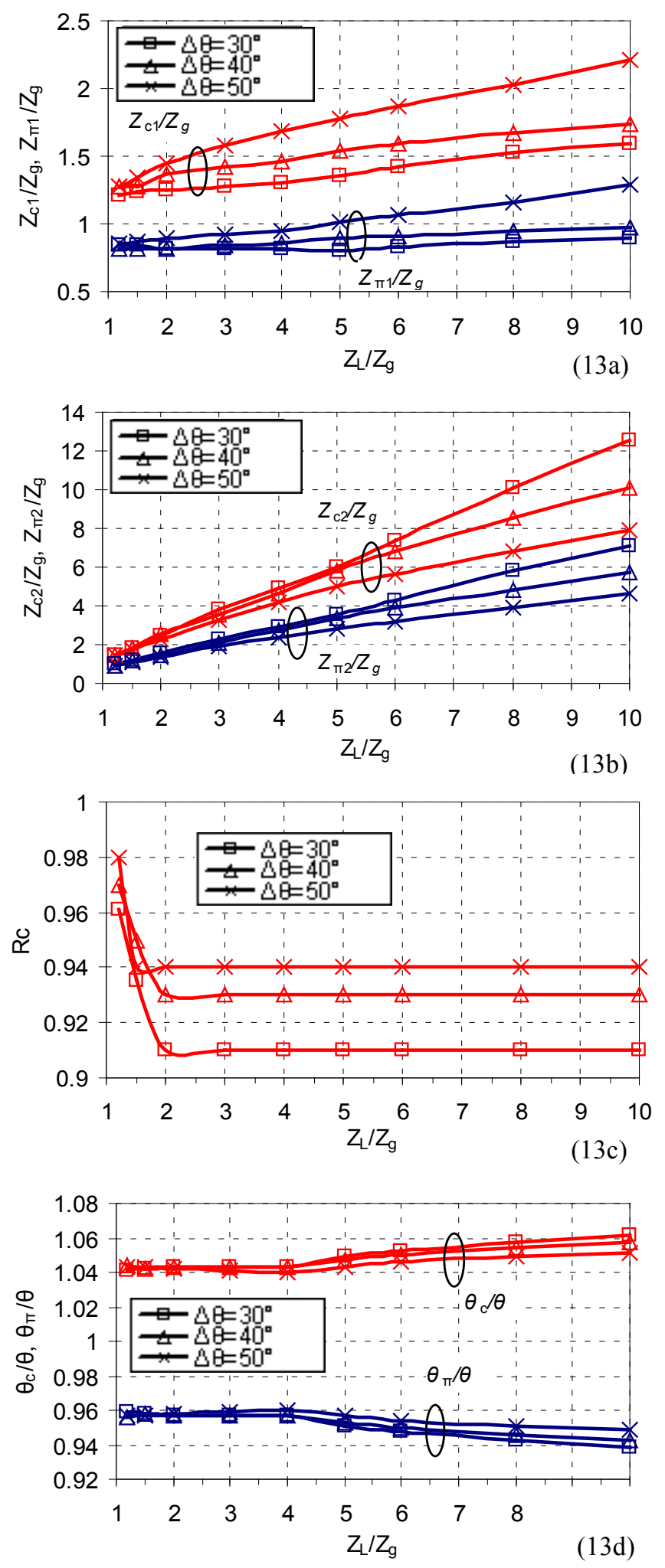


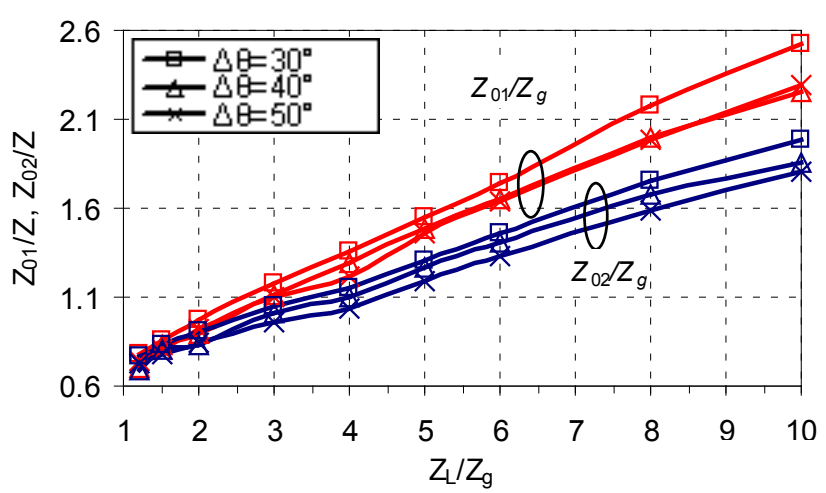

Fig. 13. Design curves for the impedance transformer in Fig. 9.

The design curve for the parameter $R_{\pi}$ is not presented here, because it can be easily found from the ratio [7]

$$
\frac{Z_{c 2}}{Z_{c 1}}=\frac{Z_{\pi 2}}{Z_{\pi 1}}=-R_{c} R_{\pi}
$$

The impedance transformer in Fig. 9 can be synthesized using the design curves in Fig. 13. It is interesting to note that the electrical lengths for both modes are essentially independent of the loading condition and can be adjusted for convenience. Furthermore, for large values of the transformation ratio $Z_{L} / Z_{g}>2, R_{c}$ does not depend on the load impedance and is only slightly dependent on $\Delta \theta$. Finally, it can be concluded from Fig. 13 that $Z_{01}$ and $Z_{02}$ can be represented in the form $Z_{01,02} / Z_{g}=Z_{00}+\mathrm{m}_{1,2} Z_{L} / Z_{g}$, where $Z_{00}$ is the constant and $m_{1,2}$ is the slope. Therefore, the transformer can be efficiently synthesized using only the parameters $Z_{c l}, Z_{c 2}$, $\mathrm{Z}_{\pi l}, \mathrm{Z}_{\pi 2}, \Delta \theta$, and $\mathrm{m}_{1,2}$.

\section{Design example}

Consider the design of a 50-100 $\Omega$ impedance transformer with the center frequency $1.1 \mathrm{GHz}$ and $\Delta \theta=40^{\circ}$.

For this 1:2 transformer the design parameters are chosen from Fig. 13 and listed in Table 1.

Table 1. Electrical parameters of the transformer.

\begin{tabular}{|c|c|c|c|c|c|c|c|c|c|c|}
\hline $\begin{array}{l}\stackrel{+0}{N} \\
\stackrel{N}{N}\end{array}$ & 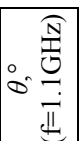 & 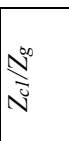 & $\begin{array}{l}N^{\infty} \\
\stackrel{3}{N}\end{array}$ & $\underset{N}{\stackrel{N}{N}}$ & $\begin{array}{l}\text { No } \\
\text { N }\end{array}$ & $\stackrel{\stackrel{N}{N}}{\stackrel{5}{N}}$ & $\begin{array}{l}\stackrel{\infty}{N} \\
\stackrel{S}{N}\end{array}$ & $\approx$ & $\stackrel{5}{\circ}$ & $\stackrel{8}{0}$ \\
\hline 2 & 90 & 1.35 & 2.5 & 0.8 & 1.5 & 0.9 & 0.8 & 0.93 & 1.042 & 0.958 \\
\hline
\end{tabular}

\begin{tabular}{|c|c|c|c|c|c|c|c|c|}
\hline $\begin{array}{l}\mathrm{C} \\
\hat{\bar{U}}\end{array}$ & $\begin{array}{l}\text { C) } \\
\text { ज̃ }\end{array}$ & $\begin{array}{l}\mathrm{C} \\
\stackrel{\mathbb{N}}{N}\end{array}$ & $\begin{array}{l}\mathrm{G} \\
\hat{\tilde{N}}\end{array}$ & $\begin{array}{l}\mathrm{C} \\
\hat{\bar{N}}\end{array}$ & $\begin{array}{l}\mathrm{C} \\
\hat{\widehat{N}}\end{array}$ & $\approx$ & 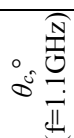 & 苞 \\
\hline 67.5 & 125 & 40 & 75 & 45 & 40 & 0.93 & 93.78 & 86.22 \\
\hline
\end{tabular}

Based on these data the physical parameters of the circuit components are synthesized. The parameters given in Table 1 correspond to a transformer based on a substrate with a dielectric constant $\varepsilon_{\mathrm{r}}=3.38$ and thickness $\mathrm{h}=0.8 \mathrm{~mm}$. The coupled line width is $1.37 \mathrm{~mm}$ for the input terminal and $0.54 \mathrm{~mm}$ for the output terminal. The gap between the coupled lines is $0.41 \mathrm{~mm}$. Microstrip line widths are $2.22 \mathrm{~mm}$ and
$2.5 \mathrm{~mm}$ for the transmission lines denoted $Z_{01}$ and $Z_{02}$ in Fig. 9, respectively. The physical length of the transformer is $43.1 \mathrm{~mm}$. Based on these physical parameters the simulation of the transformer can be performed using any freely or commercially available software circuit simulators, which contain the models for asymmetric coupled lines and microstrip transmission lines. The matching circuit design example has been fabricated and measured. A photograph of the fabricated $50-100 \Omega$ transformer is shown in Fig. 14. In this example the input transmission line is connected using an air bridge transition.

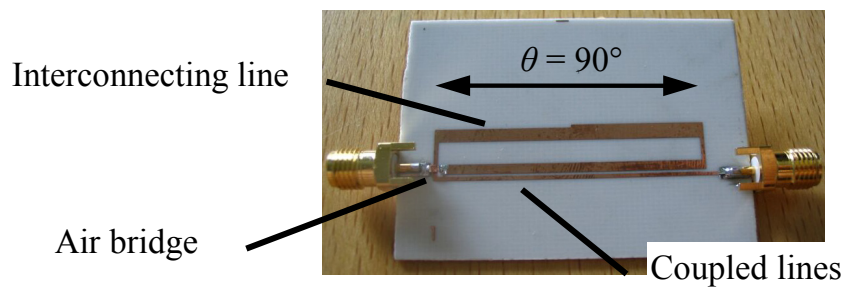

Fig. 14. Wideband quarter wavelength impedance transformer. The microwave realization of the circuit in Fig. 9.

The simulated and measured characteristics of the matching circuit are given in Fig. 15 and compared to the characteristics of the conventional quarter-wave transformer.

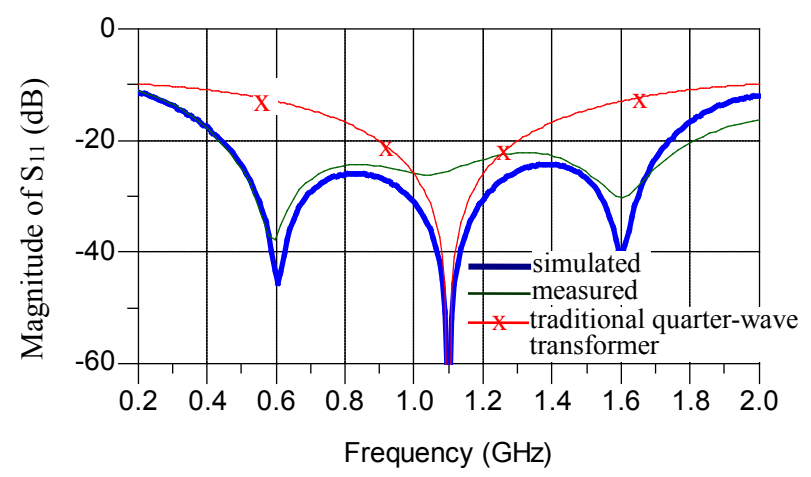

Fig. 15. Simulated and measured characteristics of the synthesized transformer; compared with the conventional quarter-wave transformer.

As it can be seen from the simulated data, three minima in the reflection coefficient spectrum of the synthesized transformer are achieved as expected. The distance between minima corresponds to $\Delta \theta=40^{\circ}$. The achieved bandwidth at $-20 \mathrm{~dB}$ reflection level is 3 times larger as compared with a standard quarter-wave matching circuit. Although the measured characteristics differ from the simulation at low magnitude of the reflection coefficient, it is deemed suitable for most practical applications. The measured fractional bandwidth for this transformer configuration is more than $120 \%$ for $-20 \mathrm{~dB}$ reflection coefficient level.

\section{CONCLUSIONS}

It is shown that coupled transmission lines is an attractive component for compact impedance transformer design. The capabilities of the coupled line transformer are extended with 
the help of different kinds of auxiliary loads, connected to diagonally opposite terminals. Using this concept different circuits have been proposed for matching real and complex loads in a narrow and wide frequency range. It is demonstrated theoretically and experimentally that it is possible to improve the fractional matching bandwidth beyond $100 \%$ at $-20 \mathrm{~dB}$ reflection level by introducing an interconnecting transmission line. Although the proposed structure is still a quarter wavelength long, it provides a three times wider operating frequency range in comparison to the traditional quarter-wave transformer.

A general model for such a configuration of the transformer was developed based on mode characteristics. This general model establishes the design curves for the impedance transformer. Based on the analysis of this model different load configurations at the free terminals are proposed resulting in improved matching characteristics of the overall circuit.

The considered examples demonstrate matching between real and complex impedances in a narrow and a wide frequency range.

\section{APPENDIX I}

A series connection of the transmission lines shown in Fig. 16 can be described as a connection of two two-port networks.

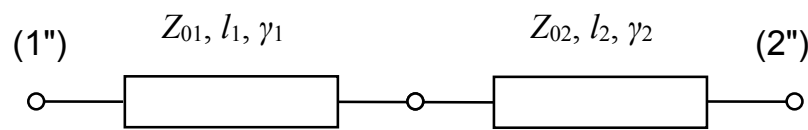

Fig. 16. Series connection of transmission lines.

The impedance matrices of the transmission lines with characteristic impedances $Z_{01}, Z_{02}$, lengths $l_{1}, l_{2}$, and propagation constants $\gamma_{1}, \gamma_{2}$ are given by

$$
\begin{aligned}
& {\left[Z^{(1)}\right]=\left[\begin{array}{ll}
Z_{11}^{(1)} & Z_{12}^{(1)} \\
Z_{21}^{(1)} & Z_{22}^{(1)}
\end{array}\right]=\left[\begin{array}{cc}
Z_{01} \operatorname{coth}\left(\gamma_{1} l_{1}\right) & \frac{Z_{01}}{\sinh \left(\gamma_{1} l_{1}\right)} \\
\frac{Z_{01}}{\sinh \left(\gamma_{1} l_{1}\right)} & Z_{01} \operatorname{coth}\left(\gamma_{1} l_{1}\right)
\end{array}\right],} \\
& {\left[Z^{(2)}\right]=\left[\begin{array}{ll}
Z_{11}^{(2)} & Z_{12}^{(2)} \\
Z_{21}^{(2)} & Z_{22}^{(2)}
\end{array}\right]=\left[\begin{array}{cc}
Z_{02} \operatorname{coth}\left(\gamma_{2} l_{2}\right) & \frac{Z_{02}}{\sinh \left(\gamma_{2} l_{2}\right)} \\
\frac{Z_{0}}{\sinh \left(\gamma_{2} l_{2}\right)} & Z_{02} \operatorname{coth}\left(\gamma_{2} l_{2}\right)
\end{array}\right] .}
\end{aligned}
$$

Impedance matrix for the overall circuit in Fig. 16 is derived using boundary conditions at the common terminal. At this terminal the voltages of two two-ports are equal, and currents are equal and oppositely directed.

Thus, impedance matrix elements are found to be:

$$
\begin{gathered}
Z_{11}^{\prime \prime}=Z_{11}^{(1)}-\frac{\left(Z_{12}^{(1)}\right)^{2}}{Z_{11}^{(2)}+Z_{11}^{(1)}}=Z_{01} \operatorname{coth}\left(\gamma_{1} l_{1}\right) \\
-\frac{Z_{01}^{2}}{\left(Z_{01} \operatorname{coth}\left(\gamma_{1} l_{1}\right)+Z_{02} \operatorname{coth}\left(\gamma_{2} l_{2}\right)\right) \cdot \sinh ^{2}\left(\gamma_{1} l_{1}\right)}, \\
Z_{12}^{\prime \prime}=Z_{21}^{\prime \prime}=\frac{Z_{12}^{(1)} Z_{12}^{(2)}}{Z_{11}^{(2)}+Z_{11}^{(1)}} \\
=\frac{Z_{01} Z_{02}}{\left(Z_{01} \operatorname{coth}\left(\gamma_{1} l_{1}\right)+Z_{02} \operatorname{coth}\left(\gamma_{2} l_{2}\right)\right) \cdot \sinh \left(\gamma_{1} l_{1}\right) \cdot \sinh \left(\gamma_{2} l_{2}\right)}, \\
\quad-\frac{\left(Z_{12}^{(2)}\right)^{2}}{\left(Z_{01} \operatorname{coth}\left(\gamma_{1} l_{1}\right)+Z_{02} \operatorname{coth}\left(\gamma_{2} l_{2}\right)\right) \cdot \sinh ^{2}\left(\gamma_{2} l_{2}\right)} .
\end{gathered}
$$

In case of transmission lines with equal electrical length $\theta / 2$ (19) can be rewritten as

$$
\begin{gathered}
Z_{11}^{\prime \prime}=Z_{01} \operatorname{coth}\left(\frac{\theta}{2}\right)-\frac{2 Z_{01}^{2}}{\left(Z_{01}+Z_{02}\right) \cdot \sinh (\theta)}, \\
Z_{12}^{\prime \prime}=Z_{21}^{\prime \prime}=\frac{2 Z_{01} Z_{02}}{\left(Z_{01}+Z_{02}\right) \cdot \sinh (\theta)}, \\
Z_{22}^{\prime \prime}=Z_{02} \operatorname{coth}\left(\frac{\theta}{2}\right)-\frac{2 Z_{02}^{2}}{\left(Z_{01}+Z_{02}\right) \cdot \sinh (\theta)} .
\end{gathered}
$$

These equations are used for the calculation of elements of the matrix $\left[Z^{\prime \prime}\right]$ in Fig. 10.

\section{APPENDIX II}

A schematical drawing showing a coupled line section with ports 2 and 4 terminated in arbitrary loads is shown in Fig. 17.

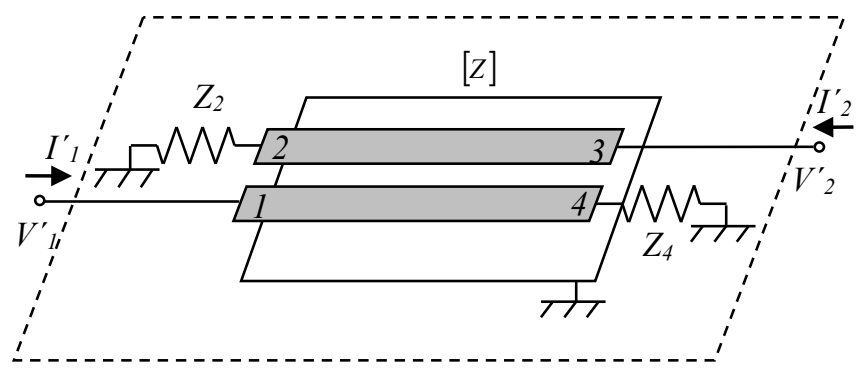

Fig. 17. 2-port circuit consisting of a coupled line section with ports 2 and 4 terminated in arbitrary impedances. The $Z$ matrix for the 4-port coupled line section is based on Eq. 1.

The impedance matrix for the 2-port based on this configuration can be written in the following form: 


$$
\left[\begin{array}{l}
V_{1}^{\prime} \\
V_{2}^{\prime}
\end{array}\right]=\left[\begin{array}{ll}
Z_{11}^{\prime} & Z_{12}^{\prime} \\
Z_{21}^{\prime} & Z_{22}^{\prime}
\end{array}\right]\left[\begin{array}{l}
I_{1}^{\prime} \\
I_{2}^{\prime}
\end{array}\right] .
$$

Where the elements of the $Z^{\prime}$-matrix can be obtained with basic circuit theory and gives

$$
\begin{aligned}
& Z_{11}^{\prime}=Z_{11}+\frac{\left(-Z_{4}-Z_{44}\right) \cdot Z_{21} \cdot Z_{12}+Z_{24} \cdot Z_{41} \cdot Z_{12}}{\Delta} \\
& +\frac{\left(-Z_{2}-Z_{22}\right) \cdot Z_{41} \cdot Z_{14}+Z_{42} \cdot Z_{21} \cdot Z_{14}}{\Delta}, \\
& Z_{12}^{\prime}=Z_{13}+\frac{\left(-Z_{4}-Z_{44}\right) \cdot Z_{23} \cdot Z_{12}+Z_{24} \cdot Z_{43} \cdot Z_{12}}{\Delta} \\
& +\frac{\left(-Z_{2}-Z_{22}\right) \cdot Z_{43} \cdot Z_{14}+Z_{42} \cdot Z_{23} \cdot Z_{14}}{\Delta}, \\
& Z_{21}^{\prime}=Z_{31}+\frac{\left(-Z_{4}-Z_{44}\right) \cdot Z_{21} \cdot Z_{32}+Z_{24} \cdot Z_{41} \cdot Z_{32}}{\Delta} \\
& +\frac{\left(-Z_{2}-Z_{22}\right) \cdot Z_{41} \cdot Z_{34}+Z_{42} \cdot Z_{21} \cdot Z_{34}}{\Delta}, \\
& Z_{22}^{\prime}=Z_{33}+\frac{\left(-Z_{4}-Z_{44}\right) \cdot Z_{23} \cdot Z_{32}+Z_{24} \cdot Z_{43} \cdot Z_{32}}{\Delta} \\
& +\frac{\left(-Z_{2}-Z_{22}\right) \cdot Z_{43} \cdot Z_{34}+Z_{42} \cdot Z_{23} \cdot Z_{34}}{\Delta}
\end{aligned}
$$

where $\quad \Delta=\left(-Z_{4}-Z_{44}\right)\left(-Z_{2}-Z_{22}\right)-Z_{42} Z_{24}$.

When $\theta=\frac{\pi}{2} ; Z_{11}=Z_{22}=Z_{33}=Z_{44}=Z_{21}=Z_{12}=Z_{34}=Z_{43}=0$.

This reduces most terms to 0 and we end up with

$$
Z_{11}^{\prime}=\frac{-Z_{2} \cdot Z_{41} \cdot Z_{14}}{\left(-Z_{4}\right)\left(-Z_{2}\right)-Z_{42} Z_{24}}
$$

But if we take $Z_{2}$ to be open-circuited $(=\infty)$ then

$$
Z_{11}^{\prime}=\frac{-\infty \cdot Z_{41} \cdot Z_{14}}{\left(-Z_{4}\right)(-\infty)-Z_{42} Z_{24}}=\frac{-Z_{41} \cdot Z_{14}}{Z_{4}}=\frac{Z_{+}^{2}}{4 Z_{4}}
$$

and similarily for the other elements of the matrix

$$
\begin{aligned}
& Z_{12}^{\prime}=Z_{13}+\frac{Z_{42} \cdot Z_{23} \cdot Z_{14}}{\left(-Z_{4}-Z_{44}\right)\left(-Z_{2}-Z_{22}\right)-Z_{42} Z_{24}}, \\
& =Z_{13}+\frac{Z_{42} \cdot Z_{23} \cdot Z_{14}}{\left(-Z_{4}\right)(-\infty)-Z_{42} Z_{24}}=Z_{13}=-\frac{j}{2} Z_{-} \\
& Z_{21}^{\prime}=Z_{31}+\frac{Z_{24} \cdot Z_{41} \cdot Z_{32}}{\left(-Z_{4}-Z_{44}\right)\left(-Z_{2}-Z_{22}\right)-Z_{42} Z_{24}}, \\
& =Z_{31}+\frac{Z_{24} \cdot Z_{41} \cdot Z_{32}}{\left(-Z_{4}\right)(-\infty)-Z_{42} Z_{24}}=Z_{31}=-\frac{j}{2} Z_{-} \\
& Z_{22}^{\prime}=\frac{\left(-Z_{4}-Z_{44}\right) \cdot Z_{23} \cdot Z_{32}}{\left(-Z_{4}-Z_{44}\right)\left(-Z_{2}-Z_{22}\right)-Z_{42} Z_{24}} . \\
& =\frac{\left(-Z_{4}\right) \cdot Z_{23} \cdot Z_{32}}{\left(-Z_{4}\right)(-\infty)-Z_{42} Z_{24}}=0
\end{aligned}
$$

Expressed as the $Z^{\prime}$ matrix for the reduced 2-port coupled lines circuit

$$
\left[Z^{\prime}\right]=\left[\begin{array}{cc}
\frac{Z_{+}^{2}}{4 Z_{4}} & \frac{-j}{2} Z_{-} \\
\frac{-j}{2} Z_{-} & 0
\end{array}\right]
$$

(2-port open-circuited impedance matrix at $\theta=\frac{\pi}{2}$ and expressed in terms of the even and odd mode impedances, $Z_{4}$ as a parameter and $Z_{2}=\infty$ O.C stub).

\section{REFERENCES}

[1] Kian Sen Ang, Chee How Lee, and Yoke Choy Leong, "Analysis and design of coupled line impedance transformers," 2004 IEEE MTT-S Int. Microwave Symp. Dig., vol. 3, pp. 1951-1954, 2004.

[2] G. Jaworski, and V. Krozer, "Broadband matching of dual-linear polarization stacked probe-fed microstrip patch antenna," Electronics Letters, vol. 40, no. 4, pp. 221-222, 2004.

[3] S. P. Liu, "Planar transmission line transformer using coupled microstrip lines," IEEE MTT-S Int. Microwave Symp. Dig., vol. 2, pp. 789-792, 1998.

[4] E.M.T. Jones and J.T. Bolljahn, "Coupled Strip Transmission Line Filters and Directional Couplers," IRE Trans. Microwave Theory \& Tech., vol. MTT-4, pp. 78-81, April 1956.

[5] V. Zhurbenko, V. Krozer, P. Meincke, "Broadband Impedance Transformer Based on Asymmetric Coupled Transmission Lines in Nonhomogeneous Medium," IEEE MTT-S Int. Microwave Symp., 2007.

[6] D. Kajfez, S. Bokka, and C. E. Smith, "Asymmetric microstrip DC blocks with rippled response," 1987 IEEE MTT-S Int. Microwave Symp. Dig., pp. 301-303, 1981.

[7] V. K. Tripathi, "Asymmetric coupled transmission lines in an inhomogeneous medium," IEEE Trans. Microwave Theory \& Tech., vol. 23, no. 9, pp. 734-739, September 1975. 